\title{
"AS MÁMOAS DOS CONCELLOS DA BAIXA LIMIA"*: APORTACIÓN AL CATÁLOGO DE MONUMENTOS TUMULARES DE LA COMARCA
}

\author{
por \\ JOSÉ Ma EGUILETA FRANCO
}

\section{1.- INTRODUCCIÓN}

A partir de los años cincuenta y tras el fallecimiento de F. López Cuevillas, la comarca orensana de A Baixa Limia queda relegada de las investigaciones dedicadas al megalitismo, excepto actuaciones aisladas de prospección y excavación y la aparición esporádica de noticias dispersas sobre hallazgos muy puntuales. Pero los trabajos de aquel investigador, fueron creando un creciente interés entre las nuevas generaciones de arqueólogos y en nosotros mismos, interés inicialmente plasmado en nuestra Tesis de Licenciatura (Eguileta Franco, 1986). Posteriormente, la investigación reiniciada desde dos de los ayuntamientos de la zona, Lobios y Muíños ${ }^{1}$, pusieron de manifiesto la existencia de diversos grupos de yacimientos repartidos por la geografía de A Baixa Limia galega.

La prospección fue uno de los aspectos fundamentales de nuestro trabajo. Por una parte, organizada en dos equipos que desarrollaron dos pro-

\footnotetext{
* Con este título queremos ofrendar un homenaje a la memoria de Florentino López Cuevillas, maestro de la Arqueología gallega.

'Que permitieron con su cooperación y aportación económica, junto con el Instituto Nacional de Empleo de Ourense y la Dirección Xeral do Patrimonio Histórico e Documental de la Xunta de Galicia, el desarrollo de los trabajos en ambos municipios.
}

"CUADERNOS DE ESTUDIOS GALLEGOS", Tomo XLI, Fascículo 106, Santiago 1993-94. 
yectos de investigación. El primero, en el Ayuntamiento de Lobios, " $O$ encoro de Lindoso e o seu entorno" $\left(1988,1989\right.$ y 1990) ${ }^{2}$ y el segundo en el de Muíños, "O Concello de Muiños e o seu marco arqueo-xeográfico", iniciado en el año 1990 y que hoy en día aún estamos llevando a cabo en campañas sucesivas. Al mismo tiempo, durante la prospección individual que realizamos y que sirvió de apoyo para un nuevo proyecto ${ }^{3}$, fueron descubiertos y registrados nuevos grupos de mámoas en los ayuntamientos integrantes total o parcialmente de A Baixa Limia.
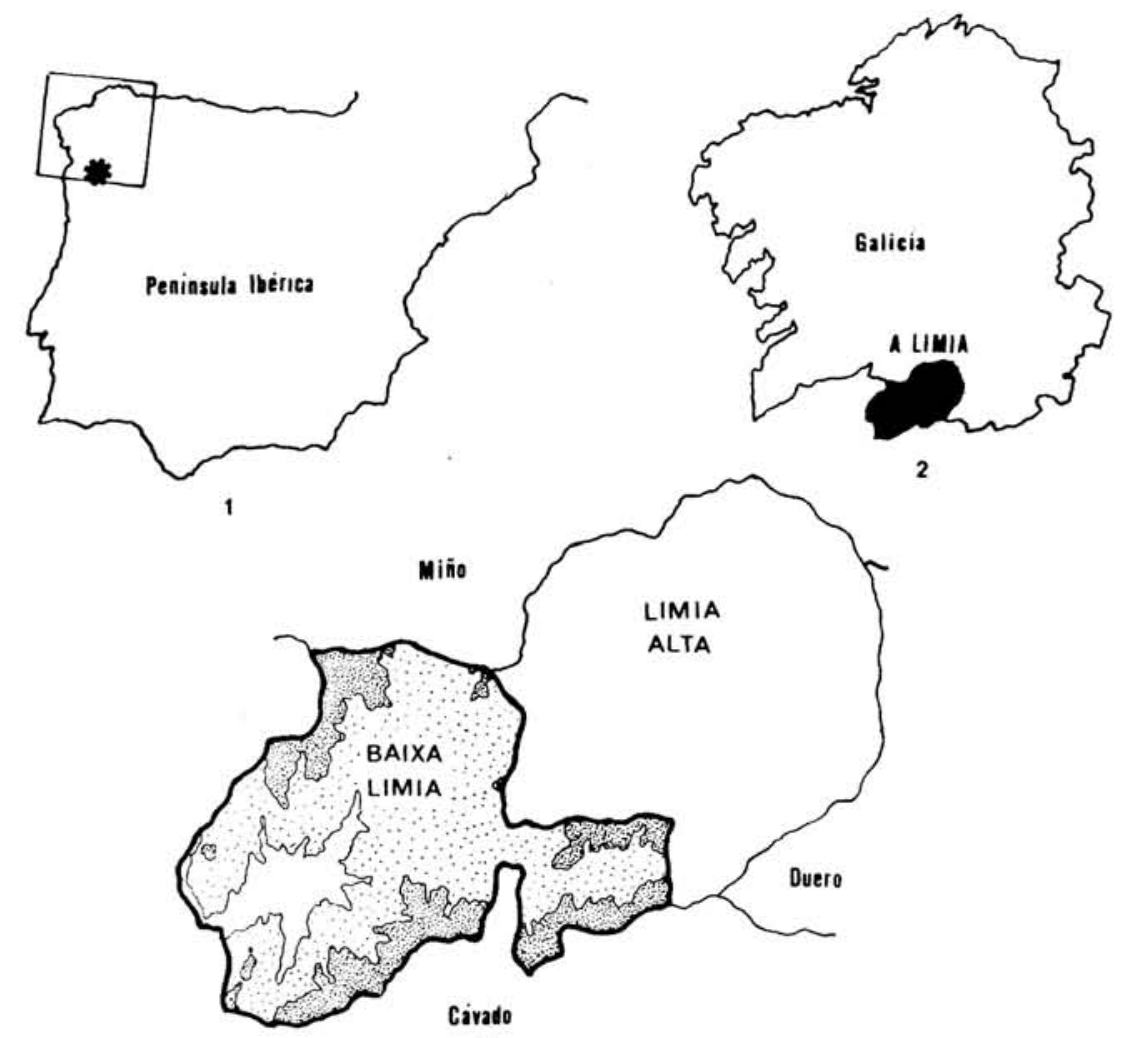

3

Fig. 1.- Localización de A Baixa Limia en el Noroeste Peninsular (1), Galicia (2) y en la comarca de A Limia (3).

${ }^{2}$ Junto con C. Rodríguez Cao (Campaña 1988) y M. Xusto Rodríguez (Campañas 1988, 1989 y 1990).

${ }^{3}$ Parcialmente plasmado en nuestra Tesis Doctoral (Eguileta Franco, 1994).

"CUADERNOS DE ESTUDIOS GALLEGOS", Tomo XLI, Fascículo 106, Santiago 1993-94. 
Ofrecemos ahora un nuevo catálogo de monumentos tumulares que, si bien no está agotado, si podemos considerar altamente representativo de la distribución de mámoas en la comarca.

\section{2.- LA COMARCA}

A Limia galega es una comarca marcadamente definida por el valle y recorrido del río Limia en el actual territorio gallego. Se extiende a lo ancho de $1250 \mathrm{~km}^{2}$ por el sector Suroccidental de la provincia de Ourense. Constituye uno de los grandes valles independientes de la Galicia Interior, limitando e individualizándose del Valle Miño-Deva-Arnoia (Norte y Oeste) y del Valle del Duero-Támega (Este) (Fig. 1). Entre las zonas Nororiental y Suroccidental, se evidencia un fuerte contraste orográfico que diferencia dos subcomarcas: A Limia Alta y A Baixa Limia respectivamente. La segunda, marco geográfico de nuestro estudio, ocupa una extensión aproximada de $580 \mathrm{~km}^{2}$ por los ayuntamiento de Lobios, Entrimo, Muíños, Lobeira, Bande y, parcialmente, Verea, Porqueira, Calvos de Randín, Baltar y Os Blancos. Durante la prospección realizada (Eguileta Franco, 1994), estructuramos el terreno de forma progresiva en las unidades estructurales siguientes:

$\left.1^{\circ}\right)$ A Baixa Limia y sus límites geográficos.

$2^{\circ}$ ) Las grandes unidades de la comarca: Valle del Salas, cadenas montañosas intermedias y la zona de sierras.

$3^{\circ}$ ) Unidades formadas por afluentes menores del río Limia y sus divisorias de aguas: valles del Caldo, Illa, Cadós, arroyo de Santa Cristina, Grou, y otras de menor entidad.

$4^{\circ}$ ) Parcelado de las unidades anteriores en cuadrículas de $1 \mathrm{~km}^{2}$, para recorrerlas a pie en una exploración minuciosa.

\section{3.- BREVE RESEÑA HISTORIOGRÁFICA SOBRE EL MEGALITISMO DE A LIMIA: ESTADO DE LA CUESTIÓN}

Desde las primeras referencias sobre la existencia de mámoas hasta la investigación actual, los estudios relacionados con el Megalitismo estuvieron definidos por unas actitudes sintomáticas de los avances de la propia investigación, con sucesivas formas de enfrentarse al Fenómeno Tumular. A Limia galega cuenta con pocos estudios y la escasa informa- 
ción se constriñe, tanto a meras alusiones, como a informes de antiguas excavaciones y otros trabajos incluidos en investigaciones arqueológicas y prehistóricas más amplias ${ }^{4}$.

En los últimos años del siglo XIX y las primeras décadas del siglo XX, contamos con las primeras referencias de R. Barros Sivelo (1875) sobre la existencia de un menhir en la Serra do Xurés, sobre las mámoas del Monte de San Cibrao y alusiones, de forma excesivamente exagerada, a las mámoas de la meseta de Santa Cristina de Monte Longo o Monte das Motas (el entorno de la Ermita do Viso) donde refiere la localización de varios centenares en un espacio de $1 \mathrm{~km}^{2}$. Por su parte, B. Fernández Alonso continúa con menciones sobre las mámoas límicas, concretamente, los "dólmenes" y mámoas de Monte das Fachas, del Monte de San Fiz o la presencia de un menhir con cazoletas en Xinzo de Limia. También señala la existencia de construcciones megalíticas en Paradela, Randín, Requiás y Maus de Salas, todas ellas en el Valle del Salas (Fernández Alonso, 1879 y 1906). En los albores del siglo XX, se publica la obra de A. Vázquez Núñez (1901) en la que, además de ofrecer unas citas más o menos precisas sobre la localización de megalitos, valora por primera vez la filiación cultural de sus constructores, los criterios de emplazamiento, el sistema constructivo de los monumentos e, incluso, el posible origen poligenista de los mismos de la mano de pueblos sedentarios. También a principios del siglo XX, J. Fortes (1901) publica el primer estudio de los megalitos del Valle del Salas, en el que se incluyen observaciones sobre la orientación de las cámaras y la mención a un ortostato decorado con pinturas en una de ellas. En su estudio sobre la relación entre mámoas y caminos, $\mathrm{M}$. Díaz Sanjurjo (1904) menciona la mámoa de Alto do Vieiro, proponiendo un nuevo patrón de asentamiento: los pasos de las divisorias de aguas.

Con la Xeración Nós, y tras la presencia en Galicia de H. Obermaier, los estudios prehistóricos gallegos alcanzan el contenido científico que se está implantando en otras áreas de la Península Ibérica (Obermaier, 1923). Desde los primeros años de la década de los veinte, los esfuerzos orientados hacia el estudio del megalitismo se acometieron dentro de una tentati-

\footnotetext{
${ }^{4}$ Véase, por ejemplo, RODRÍGUEZ CASAL, A.A., 1990, O Megalitismo. A primeira arquitectura monumental de Galicia. "Biblioteca de Divulgación", Serie Galicia, 4, Santiago de Compostela; 1991, "O Megalitismo galego: a documentación arqueográfica". En Galicia Historia, I, Prehistoria e Historia Antiga, Hércules Ediciones, Santiago de Compostela.
} 
va de superar los aspectos descriptivos a través de las actividades de prospección y excavación. Entre los estudiosos despuntan F. Maciñeira, que centró sus estudios en el área septentrional gallega ${ }^{5}$, y F. López Cuevillas, más vinculado al sector meridional y que será el principal impulsor de los estudios prehistóricos en Galicia. Entre los trabajos de prospección y excavación de mámoas es necesario destacar los estudios sobre Monte das Motas (López Cuevillas, 1923 y 1925a), Serra de Leboreiro (López Cuevillas, 1925a), San Cibrao, A Mota Grande y a Mota Pequena de Monte Albán (López Cuevillas, 1925b) o las mámoas del Valle del Salas (López Cuevillas, 1927a). También en ese mismo año, inicia F. López Cuevillas sus "papeletas" sobre o Monte das Motas, Serra de Leboreiro, Monte Grande, Monte das Fachas, Oleiro de Antas, San Cibrao, Monte Albán y Valle del Salas (López Cuevillas, 1927b). A estas actividades debemos añadir el estudio de las colecciones de materiales (López Cuevillas, 1930), las síntesis de índole prehistórica (López Cuevillas y Bouza Brey, 1931) o los estudios combinados con la Etnología (López Cuevillas y Lorenzo Fernández, 1930).

De la mano de otros investigadores vinculados a la Xeración Nós, nos llegan nuevas noticias sobre los monumentos tumulares límicos. Así, $\mathrm{H}$. García (1922), que menciona la existencia de un dolmen cerca de Entrimo, o Xurxo Lorenzo y L. Fariña (1933) que citan las mámoas de la Serra de Leboreiro (Lorenzo y Fariña, 1933) y A. Couceiro Freijomil (1937), con una sucinta mención sobre las mámoas de A Lampaza. A finales de la década de los treinta, en la obra de G. Leisner (1938) sobre el Megalitismo de Galicia y del Norte de Portugal, encontramos referencias arquitectónicas sobre las mámoas del Valle del Salas y de Calvos de Randín.

La creación del "Instituto Padre Sarmiento de Estudos Galegos"(1944)

\footnotetext{
${ }^{5}$ Véase, por ejemplo, MACIÑEIRA, F., 1929, "Notable grupo de círculos líticos y túmulos dolménicos en la cuenca superior del río Eume". Arquivos do Seminario de Estudos Galegos, 2; 1935, "La distribución de las estaciones prehistóricas ortegalesas y sus características". Boletin de la Real Academia Gallega, XXII, A Coruña; 1941, "El vaso campaniforme y condiciones de sus intactos yacimientos en la estación de Puentes del Cabo Ortegal". Atlantis, "Actas y Memorias de la Sociedad Española de Antropología, Etnografía y Prehistoria", XVI; 1943 "Túmulos prehistóricos. Inventario descriptivo de los doscientos ochenta y seis túmulos prehistóricos hasta ahora descubiertos en la avanzada comarca del Cabo Ortegal". Boletín de la Real Academia Gallega, XXIII, A Coruña (en varios apartados y páginas); 1947, Bares. Puerto hispánico de la primitiva navegación occidental. Centro Superior de Investigaciones Científicas. Santiago de Compostela.
}

"CUADERNOS DE ESTUdIOS GALLEGOS", Tomo XLI, Fascículo 106, Santiago 1993-94. 
representa otro de los grandes hitos cronológicos para la investigación en Galicia. En el Sur, F. López Cuevillas es el máximo exponente de una madurez investigadora que impregna los estudios relacionados con las mámoas, ya sean trabajos de síntesis (López Cuevillas, 1948, 1955, 1959 y 1973) o excavaciones, como la de Galez (López Cuevillas y Lorenzo Fernández, 1958). Por esos mismos años, la prolífica labor de G. y V. Leisner dará un nuevo impulso a la investigación, entre otros, de los megalitos gallegos (Leisner G. y V. 1959). No queremos olvidar la mención al posterior estado de la cuestión que, a partir de 1952, nos ofrece J. Taboada Chivite (1973).

A finales de los sesenta, la Arqueología gallega recibirá unha nueva inyección de fuerza con la creación del "Seminario de Arqueoloxía" en la Universidad de Santiago de Compostela (1968). Bajo el magisterio de A. Balil Illana y C. Alonso del Real y Ramos, una generación de arqueólogos y prehistoriadores, entre ellos J.C. Sierra Rodríguez, A.A. Rodríguez Casal, J.M. Vázquez Varela, M.C. García Martínez, etc., incidieron en novedosos aportes a la investigación tumular gallega. Sin embargo, A Limia permaneció al margen, tanto de programas como de actuaciones puntuales por parte de este grupo de arqueólogos. La investigación relacionada con los yacimientos tumulares en el Suroeste Ourensano se paraliza casi totalmente, excepto la "reexcavación" de las mámoas $\mathrm{N}^{\circ} 1$ y No 3 de Maus de Salas por J. Ferro Couselo (1972) y de la prospección multidisciplinar centrada en el Valle del Salas (VV.AA., 1980), desgraciadamente inédita. Contamos con un último trabajo de A. Rodríguez Colmenero (1971) en la Serra de Larouco, límite oriental de A Limia.

Será una nueva generación de investigadores la que retome la investigación, si bien de forma parcial, como R. Fábregas Valcarce y F. de la Fuente Andrés (Fábregas Valcarce y Fuente Andrés, 1988; Fábregas Valcarce, 1993) que estudian las cerámicas y los materiales líticos de A Mota Grande y A Mota Pequena de Monte Albán, de la M7 do Monte das Motas, y de Maus de Salas. Posteriormente, nosotros mismos, en un trabajo que presentamos como Tesis de Licenciatura (Eguileta Franco, 1986) sobre los ajuares de las mámoas del Valle Medio del Miño y de A Limia, estudiamos los materiales procedientes de los túmulos límicos (Eguileta Franco, 1987).

En este mismo momento, se inicia una serie de actividades programadas de elaboración de Cartas Arqueológicas en las que se incluyen mapas de distribución de monumentos tumulares. Llevamos a cabo prospecciones junto con C. Rodríguez Cao y M. Xusto Rodríguez, en Lobios, donde 
se catalogan las mámoas de S. Bieito, Anta do Couto e Portela de Arcas (Eguileta Franco, Rodríguez Cao y Xusto Rodríguez, 1991). Contamos, además, con la de C. Rodríguez Cao en Bande, donde se registran las mámoas de A Cañota y Nogueira (Rodríguez Cao, 1990) y nosotros mismos en Muíños y en la totalidad de la comarca, con catalogación de las mámoas de Corga de Arcas, Monte de Pazó, Outeiro de Cachofás, Monte de Reparada, O Nicho, A Lampaza, A Anta, Catrocamiños, Reboral, Poza da Lobada, Outeiro de Cavaladre, A Lagoa, A Portela de Pitoes, Padín, Fonte Santa, A Lagoa, Os Aceviñeiros, Penedos da Canteira, Outeiro de Augas, A Formiga, Queguas, etc. (Eguileta Franco, 1991 y 1994), prospecciones que incluyeron parcialmente actividades de excavación.

\section{LAS MÁMOAS}

\subsection{Inventario de monumentos}

Durante los trabajos de prospección llevados a cabo hasta el momento en la comarca, fueron catalogadas las mámoas y grupos de monumentos tumulares siguientes (Fig. 2):

1. PORTELA DAS ARCAS, Lobios (Eguileta Franco, Rodríguez Cao y Xusto Rodríguez, 1991).

2. ANTA DO COUTO, Lobios (Eguileta Franco, Rodríguez Cao y Xusto Rodríguez, 1991).

3. CORGA DE ARCAS, Muíños (Eguileta Franco, 1991).

4. MONTE DE PAZO, Muíños (Eguileta Franco, 1991).

5. OUTEIRO DE CACHOFAS, Muíños (Eguileta Franco, 1991).

6. MONTE DE REPARADE, Muíños (Eguileta Franco, 1991).

7. O NICHO, Muíños (Eguileta Franco, 1991).

8. A LAMPAZA, Muíños (Eguileta Franco, 1991).

9. A ANTA, Muíños (Eguileta Franco, 1991).

10. CRUCE DE XERMEADE, Muíños (Eguileta Franco, 1991).

11. CATRO CAMIÑOS, Muíños (Eguileta Franco, 1991).

12. O REBORAL, Muíños (Eguileta Franco, 1991).

13. VEIGA DE MAUS DE SALAS, Muíños (López Cuevillas 1927a; VV.AA., 1980; Eguileta Franco, 1991).

14. VEIGA DE REQUIAS, Muíños (VV.AA., 1980; Eguileta Franco, 1991)

15. PADIN, Muíños (Eguileta Franco, 1991).

16. SAMPEDRO, Tourem-Portugal (Eguileta Franco, 1991).

"CUADERnOS DE eSTUdios GALLEGOS", Tomo XLI, Fascículo 106, Santiago 1993-94. 


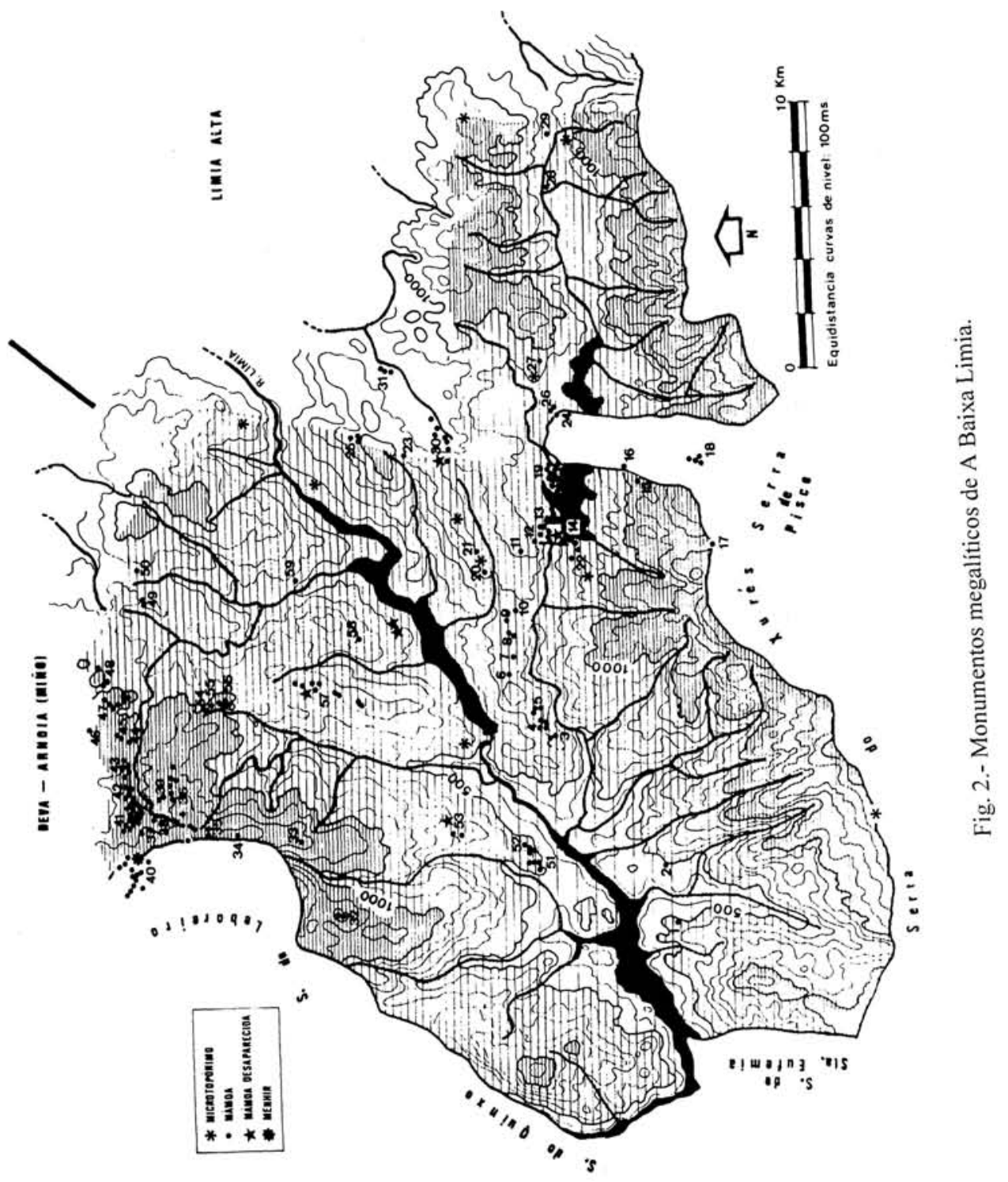


17. PORTELA DE PITÕES, Muíños (Eguileta Franco, 1991).

18. A MOURELA, Tourem-Portugal (Oliveira Jorge, V., 1982).

19. OUTEIRO DE CAVALADRE, Muíños (Eguileta Franco, 1991).

20. PORQUEIROS, Muíños (VV.AA., 1980; Eguileta Franco, 1991).

21. AGROCHAO, Muíños (Eguileta Franco, 1991).

22. MUNIXEI, Muíños (Eguileta Franco, 1991).

23. POZA DE LEVADA, Muíños (Eguileta Franco, 1991).

24. MARCO No 95, Calvos de Randín (Eguileta Fanco, 1991).

25. A LAGOA, Porqueira (Eguileta Franco, 1991).

26. OUTEIRO DAS MOAS, Calvos de Randín (VV.AA., 1980; Eguileta Franco, 1991).

27. OUTEIRO DE PEDREIRA, Calvos de Randín (Eguileta Franco, 1991).

28. FONTE SANTA, Baltar (Eguileta Franco, 1991).

29. MEDEXORXE, Baltar (Eguileta Franco, 1991).

30. A MOURA, Muíños-Calvos de Randín (López Cuevillas y Lorenzo Fernández, 1930; VV.AA., 1980; Eguileta Franco, 1991).

31. A CHAMUSCADA, Calvos de Randín (Eguileta Franco, 1991).

32. QUEGUAS, Entrimo (Eguileta Franco, 1994).

33. MOURISCA DE ARRIBA, Lobeira (Eguileta Franco, 1994).

34. MOTA DA CABREIRA, Lobeira (Eguileta Franco, 1994).

35. GRAMA DO CORNO DOURADO, Lobeira (Eguileta Franco, 1994).

36. SERRA DAS MOTAS, Lobeira (López Cuevillas, 1925a; Eguileta Franco, 1994).

37. SALGUEIRO SECO, Verea (Eguileta Franco, 1994).

38. OESTE DE OUTEIRO DAS MOS, Lobeira (Eguileta Franco, 1994).

39. OUTEIRO DAS MOS, Lobeira (Eguileta Franco, 1994).

40. OUTEIRO DO FERRO-PENAGACHE, Castro Leboreiro (Portugal) - Verea (Rodríguez Cao, 1993; Eguileta Franco, 1994).

41. PENEDOS DA CANTEIRA, Verea (Eguileta Franco, 1994).

42. OS ACEVIÑEIROS, Verea (Eguileta Franco, 1994).

43. FONTE DO BIDO, Verea (Eguileta Franco, 1994).

44. A MOTA, Verea (Eguileta Franco, 1994).

45. AS CATRO CRUCES, Verea (Eguileta Franco, 1994).

46. PEDRA DA ARCA, Verea (Eguileta Franco, 1994).

47. OUTEIRO DE AUGAS, Bande (Eguileta Franco, 1994).

48. A MOA, Bande (Eguileta Franco, 1994).

49. MONTE DE CALVOS, Bande (Eguileta Franco, 1994).

50. O VIEIRO, Bande (Díaz Sanjurjo, 1904; López Cuevillas, 1927b; 
López Cuevillas y Bouza Brey, 1931).

51. CHAN DE ANDRE, Entrimo (López Cuevillas y Lorenzo Fernández, 1958).

52. CHAIRA DE SAN FIZ, Entrimo (López Cuevillas y Lorenzo Fernández, 1958).

53. SAN BIEITO, Lobios (Eguileta Franco, Rodríguez Cao y Xusto Rodríguez, 1991).

54. OS PEDROUZOS, Bande (Eguileta Franco, 1994).

55. PENEDOS DA CHAN, Bande (Eguileta Franco, 1994).

56. A FORMIGA, Bande (Eguileta Franco, 1994).

57. MONTE DAS MOTAS, Lobeira (López Cuevillas, 1923 y 1925a; Eguileta Franco, 1994).

58. NOGUEIRA, Lobeira (Rodríguez Cao, 1991).

59. A CAÑOTA, Bande (Rodríguez Cao, 1991).

4.2. Características generales de los monumentos tumulares de A Baixa Limia

A partir de los análisis realizados en función de las diversas características morfotipológicas y métricas de los monumentos, ofrecemos a grandes rasgos unos patrones constructivos y regularidades arquitectónicas de las mámoas catalogadas. Debemos, sin embargo, tener en cuenta que parte de esas valoraciones debemos contemplarlas con cautela. De esta forma, con respecto a las cámaras, ofrecemos una clasificación tipológica, siempre con un criterio de provisionalidad que deriva de varios factores, principalmente la escasez de excavaciones, el alto grado de destrucción que sufren muchos monumentos y la dificultad de clasificar las cámaras en superficie con fiabilidad. Así, y teniendo en cuenta esa eventualidad, agrupamos las cámaras en las formas siguientes (Fig. 3):

1) Cámaras poligonales simples:

a) Regulares y abiertas, a veces de gran tamaño (M1 de Veiga de Requiás $/ \mathrm{N}^{\mathrm{o}} 1$ ), con dimensiones medias (M1 de Monte das Motas / $\mathrm{N}^{\circ} 2$ ) y pequeñas (Pena de Anduriña / $\mathrm{N}^{\circ} 3$ ).

b) Alargadas cerradas y quizá, también abiertas (M4 de Serra das Motas / No 4, M1 de Munixei / No 5 y M6 de A Moura / Nº 6).

2) Cámaras provistas de corredor:

a) Con espacio interior indiferenciado en planta y alzado y estrechamiento progresivo del recinto funerario (M1 de Veiga de Maus de Salas / $\mathrm{N}^{\mathrm{o}}$ 7).

b) Cámaras poligonales con corredor que estrecha el acceso al recinto,

"CUADERNOS DE ESTUDIOS GALLEGOS", Tomo XLI, Fascículo 106, Santiago 1993-94. 


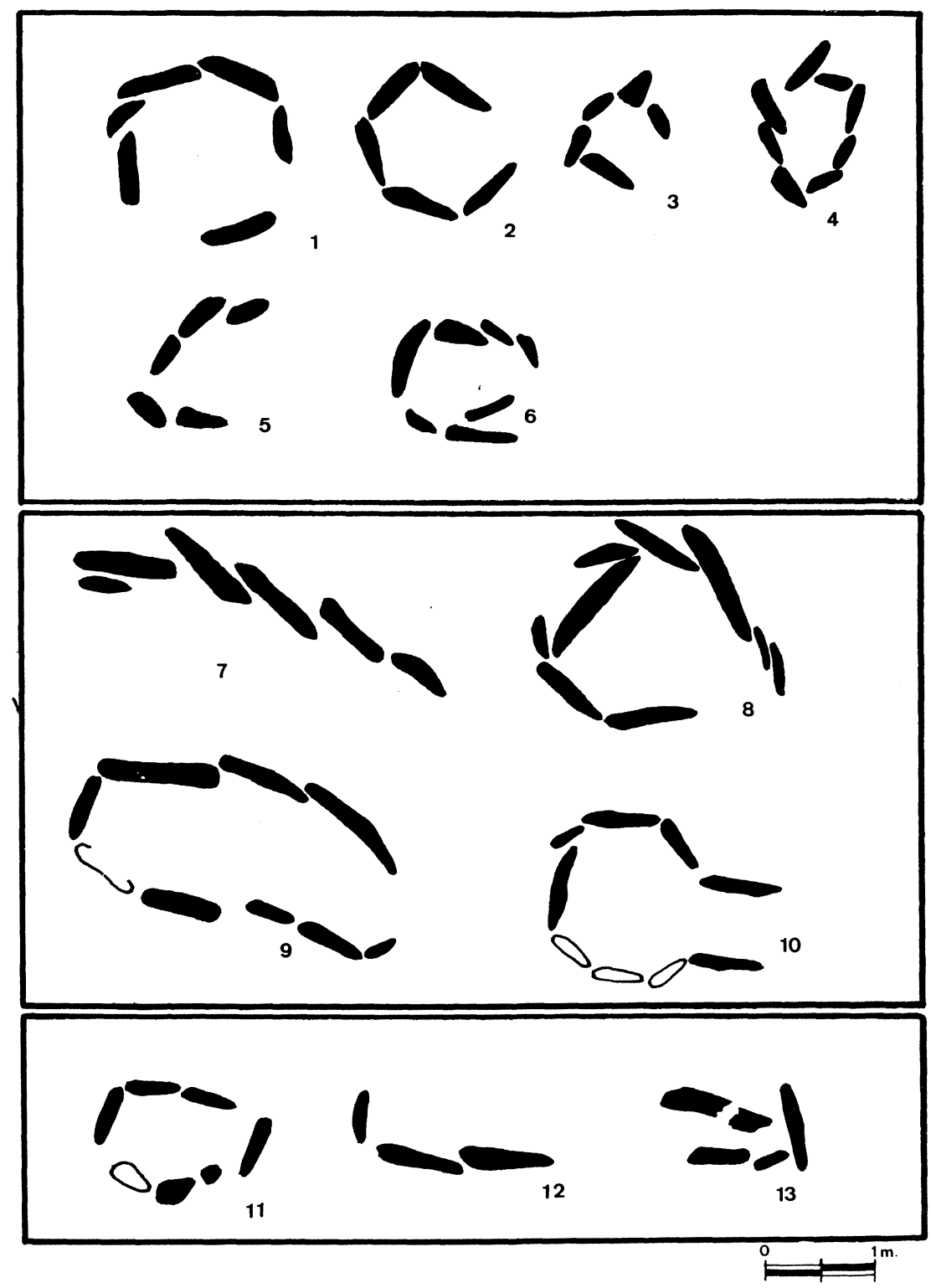

Fig. 3.- Cuadro tipológico de las cámaras megalíticas de A Baixa Limia (Eguileta Franco, 1994). 
con espacio interior diferenciado en alzado (M8 de Veiga de Maus de Salas o "A Casiña da Moura" / No 8).

c) Cámara con tendencia rectangular y recinto funerario diferenciado en alzado (M1 de Outeiro de Cavaladre / No 9).

d) Cámara poligonal con vestíbulo largo de acceso y recinto interior diferenciado en planta y alzado (M4 de Serra das Motas / No 10).

3) Pequeñas cistas restangulares:

a) Cámaras cistoides irregulares y con ortostados asimétricos y desiguales en formas y tamaños (M5 de Outeiro de Cavaladre / $\mathrm{N}^{0} 11$ ).

b) Cámaras rectangulares (M4 de Veiga de Requiás / No 12 y M10 de Outeiro de Cavaladre / $\mathrm{N}^{\circ} 13$ ).

Estas tipologías denotan una gran variabilidad constructiva en formas y tamaños, formas no obstante encuadrables en las clasificaciones ofrecidas hasta ahora para el Noroeste Peninsular (cfr. por ej. Leisner, G., 1938, López Cuevillas, 1973 o Rodríguez Casal ${ }^{6}$ ).

Por otra parte, los diámetros mayores de los túmulos muestran, a primera vista, una diversidad que oscila entre valores próximos a los $7.50 \mathrm{~m}$. y los $54 \mathrm{~m}$. Sin embargo, los dos son excepcionales, ya que parece detectarse una tendencia hacia varios patrones constructivos en el tamaño de las masas tumulares. En este sentido, las mayores coincidencias de medidas diametrales oscilan entre los $13 \mathrm{~m}$. y los $16 \mathrm{~m}$. Con menor representación, aunque con una frecuencia a considerar, se observan también patrones en torno a los $20 \mathrm{~m}$. y a los próximos a los $10 \mathrm{~m}$. En función de estos datos, debemos considerar casi excepcionales los diámetros con tamaños superiores a los $25 \mathrm{~m}$. y, totalmente excepcionales, los tres monumentos que llegan, en dos casos a los $40 \mathrm{~m}$. de diámetro (A Mota y Fonte Santa) y, en uno, a los de $52 \mathrm{~m}$. (A Mota Grande o M1 de Outeiro do FerroPenagache).

Las masas tumulares presentan en planta una forma dominante con cierto alargamiento tendente a la elipse suave, dado el predominio de la desigualdad de medidas entre cada uno de sus diámetros (Norte-Sur y Oeste-Este). Existe una preferencia casi total por la construcción de las masas tumulares suavemente alargadas en sentido Oeste-Este. Las geométricamente circulares (con la misma dimensión en los dos diámetros), tienen una representación relativamente pequeña. Sin embargo, los

\footnotetext{
${ }^{6}$ Véase Nota $n^{\circ} 4$
} 
Indices de Alargamiento del Túmulo ${ }^{7}$, muestran la preferencia por las plantas próximas al círculo, sumando entre los alargamientos con valores bajos y las "geométricamente" circulares, más de las tres cuartas partes del total de los monumentos. También los Indices de Alargamiento del Túmulo (I.A.T.) denotan la rareza y excepcionalidad de las masas tumulares en forma de elipse muy exagerada o excesivamente alargada, representada solamente en dos monumentos. Esta tendencia al alargamiento en sentido Oeste-Este no obedece, en la mayoría de los casos, a una necesidad constructiva relacionada con las tipologías de las cámaras, a no ser los leves indicios que nos presentan los pocos datos con que contamos y relacionados con monumentos que acogen en su interior cámaras provistas de corredor. Así pués, la preferencia por el alargamiento tumular en sentido Oeste-Este, debe responder a otros motivos diferentes de los condicionantes arquitectónicos impuestos por las formas de las cámaras ${ }^{8}$.

Los monumentos registrados nos ofrecen un número mayoritario de volúmenes relativamente reducidos (con valores inferiores a los $150 \mathrm{~m}$. cúbicos), que se corresponden con alturas predominantemente medias y bajas (inferiores a los $120 \mathrm{~cm}$. en el punto más alto de la masa tumular conservada). Parece observarse una ligera tendencia general al incremento del volumen de los monumentos en función del factor altitud, aunque sin una distribución en la relación volumen/altitud lo suficientemente clara para que atestigüe este indicio. Tampoco parece existir una relación directa entre los volúmenes de los monumentos y las tipologías de las cámaras, a no ser la tendencia entre alturas bajas y volúmenes pequeños detectada en las mámoas que encierran pequeñas cistas rectangulares.

Las masas tumulares de las mámoas de la comarca están formadas, fundamentalmente, por tierra limpia a la que se superpone una coraza de piedras $^{9}$, o bien por un amontonamiento de tierra y piedras de pequeño y mediano tamaño que a veces semeja un cairn. No contamos con datos

${ }^{7}$ I.A.T., dado por el cociente entre las dimensiones de los diámetros Oeste-Este y Norte-Sur (Eguileta Franco, 1994).

${ }^{8}$ Uno de ellos pudiera estar relacionado con el ritual, quizá presente en toda la composición arquitectónica del monumento, ya que las formas de las cámaras si mantienen el Oriente como punto de referencia: las poligonales para la situación de la puerta de acceso; en las provistas de corredor, este elemento comunica la cámara con el Naciente; las cistas rectangulares se orientan también sobre este eje Oeste-Este, en sentido longitudinal.

${ }^{9}$ Con distribución más o menos regular y que cubre los túmulos total o parcialmente. 
suficientes, con la excepción de observaciones ocasionales, para considerar una posible regularidad de anillos líticos u otros elementos arquitectónicos en el interior de los túmulos.

Sí pudimos comprobar en dos de las excavaciones realizadas, la existencia de unas placas pétreas de sellado que cierran casi herméticamente los intersticios que van quedando entre los ortostatos. Aquellas placas se iban colocando a medida que la tierra apilada durante la construcción de la masa tumular va ganando altura.

En la relación espacial cámara/masa tumular, se observan varias posibilidades, desde la ocultación de las antas en el interior del túmulo, hasta la exagerada elevación de éstas sobre la masa tumular. Sin embargo, la construcción más frecuente es aquella en la que la losa de cobertura de la cámara alcanzaría una altura similar a la superficie del túmulo en el lugar central del monumento. No creemos observar en la comarca una relación directa entre las posiciones de las cámaras con respecto a las masas tumulares y una cronología, periodización o evolución arquitectónica derivada de la tipología de esas cámaras. Lo mismo que la ocultación total está comprobada en algunos monumentos de gran tamaño con cámaras poligonales y en una provista de corredor, éstas también aparecen de forma muy frecuente y regular alcanzando la superficie del túmulo. Así también, las pequeñas cistas rectangulares se reparten entre las que sobresalen notoriamente del túmulo y las que $\mathrm{su} / \mathrm{s}$ losa/s de cobertura llegaría/n aproximadamente hasta la altura superficial del monumento.

\section{5.- BREVE COMENTARIO ACERCA DE ALGUNAS REGULA- RIDADES DETECTADAS EN EL EMPLAZAMIENTO DE LAS MÁMOAS EN A BAIXA LIMIA}

\subsection{Sobre la orografía}

Con respecto a la orografía, en la comarca se constata una fuerte tendencia al emplazamiento de las mámoas en las divisorias de aguas principales (cerca del 75\%), evitando la mayoría de ellas los talwegs altitudinalmente bajos (Fig. 2). Otro de los emplazamientos importantes, aunque con menor entidad, es el de valles abiertos (que agrupan casi el $25 \%$ de las mámoas), ofreciendo una alternativa a la posición predominante de divisoria, posición quizá provodada por la accidentada orografía de las sierras que limitan los valles de Ponte Maior y Salas. El factor altitud puede condicionar, junto con otros, la opción de emplazamiento de 
valle, poniéndose de manifiesto el abandono de valles altitudinalmente inferiores.

Las portelas, lugares claves por facilitar el acceso y permitir el tránsito entre grupos de monumentos con distintas posiciones orográficas, aparecen ocupadas por una o varias mámoas. Creemos observar, además, una relación directa entre la monumentalidad de las mámoas y la señalización de los lugares de paso principales y de aquellos otros donde la especial topografía del relieve (como la diferencia de rasantes o la inflexión de pendientes) dificulta la comunicación visual entre espacios ocupados por sepulturas tumulares más o menos distantes.

También los relieves aplanados son lugares preferentes de edificación de las mámoas, actuando como denominador común prioritario en las diversas opciones de los emplazamientos, tanto en las divisorias de aguas como en los fondos y laderas de los valles y en las portelas, ocupando el espacio más llano de todos y cada uno de los entornos (Fig. 4). En las concentraciones de monumentos, las mámoas edificadas en ladera se corresponden con sepulturas marginales, bien desde el punto de vista cronológico, bien desde criterios monumentales, es decir, con menor volumen de masa tumular.

Cuando los monumentos se emplazan en posiciones de "visibilidad atenuada" (como es el caso de los fondos de valle), presentan volúmenes

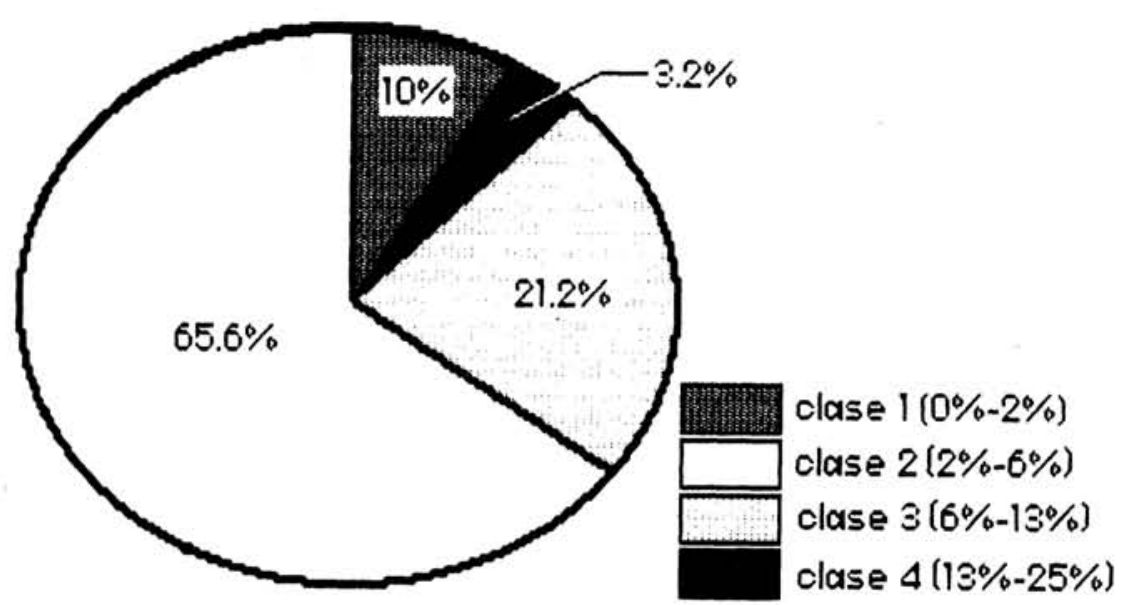

Fig. 4.- Porcentaje de las mámoas catalogadas según las pendientes topográficas.

"CUADERNOS DE ESTUdios GALLEGOS", Tomo XLI, Fasciculo 106, Santiago 1993-94. 
que les confieren una monumentalidad realzada o añadida, razón por la que esos emplazamientos debieron ser obligados o predeterminados desde los criterios de selección espacial de sus constructores ${ }^{10}$.

\subsection{Sobre el sustrato geológico}

Entre los monumentos registrados, existe una clara preferencia por el granito como material constructivo de las cámaras. Tan solo ocasionalmente se recurre al material del sustrato si este no es granítico, lo que nos lleva a considerar el uso de otros materiales diferentes (esquisto y migmatitas nebulíticas) como puntual y esporádico. Existen ejemplos en los que un mismo grupo de mámoas se extiende por dos sustratos diferentes. Cuando uno de ellos es granítico, esta será la roca usada en la construcción de la sepultura.

En A Baixa Limia el granito, que reune buenas propiedades mecánicas para la construcción de losas, es la roca predominante. Incluso en sustratos formados por materiales diferentes (migmatitas nebulíticas y esquistos) aflora con frecuencia, ofreciendo muchos puntos repartidos por la zona en los que su extracción es fácil. Es por ello que el sustrato geológico parece, teniendo en cuenta la preferencia de los constructores por el granito como materia prima para la elaboración de los ortostatos, mas que condicionar, facilitar la ubicación de gran parte de los monumentos catalogados, sobre todo los construidos sobre los esquistos. A juzgar por las relaciones mámoas-sustrato geológico, los monumentos tumulares semejan distribuirse sobre los tres grupos de rocas principales de la comarca en una proporción similar a la superficie que cada una de ellas ocupa en la misma (Fig. 5). No obstante, esta primera apreciación es matizable, ya que los esquistos se distribuyen por el entorno de las rocas graníticas, que afloran con discontinuidad entre el sustrato pizarroso ${ }^{11}$. También en el

\footnotetext{
${ }^{10}$ Esta circunstancia la detectamos en la comarca en relación con caminos de fondo de valle, tal y como ocurre con las mámoas de A Chamuscada y A Moura, alineadas a lo largo de un camino que comunicaría A Limia Alta con A Baixa Limia.

"Debemos hacer, sin embargo, una observación al respecto: estratigraficamente, las rocas graníticas se sitúan bajo los esquistos y migmaticas. Por causa de los movimientos tectónicos, el granito irrumpe en cuña a la superficie, perforando los materiales que se le superpongan y haciéndolos resbalar en placas por sus ccstados. Consecuentemente los granitos alcanzan las zonas más elevadas, lugar preferente para los emplazamientos de las mámoas. Esto es lo mismo que decir que la relación mámoas-granito es efecto indirecto de la tectónica que, posteriormente, hizo más fácil la obtención de la materia prima para la construcción de los megalitos.
}

"CUADERNOS DE ESTUdIOS GALLEGOS", Tomo XLI, Fascículo 106, Santiago 1993-94. 


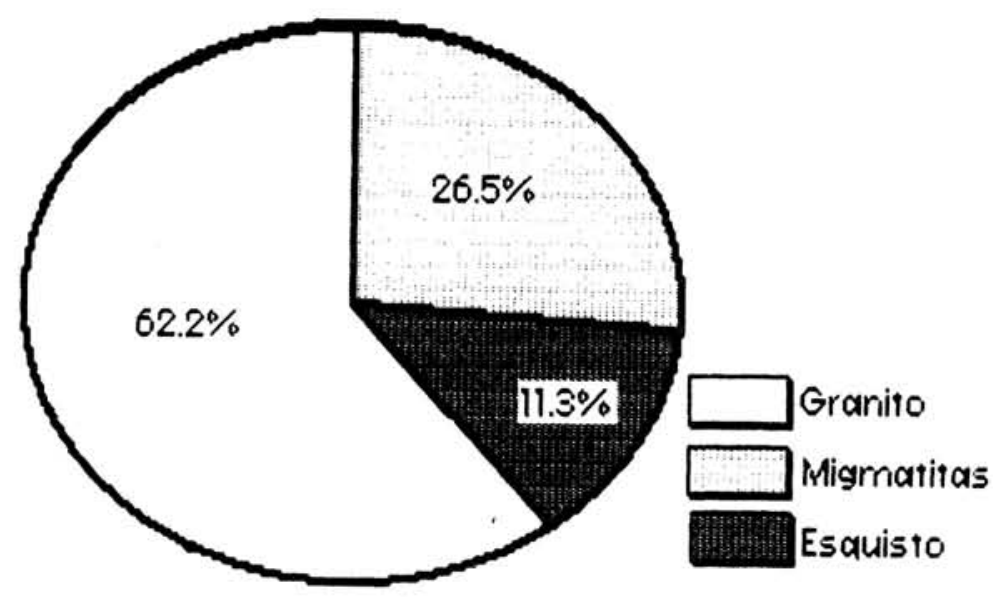

Fig. 5.- Porcentaje de las mámoas catalogadas sobre los tres principales sustratos rocosos de A Baixa Limia.

sustrato migmatítico, una parte importante de los monumentos se emplaza en las inmediaciones de los afloramientos graníticos ${ }^{12}$.

En aquellos lugares en los que la facilidad y proximidad de extracción de losas se combina con otras características de supuestas propiedades óptimas de habitabilidad (tierras aptas para el cultivo, praderas naturales, abundancia de recursos hídricos, etc.), se localizan las grandes concentraciones de monumentos (como en el Valle del Salas y en las penillanuras superiores y estribaciones inmediatas de la Serra de Leboreiro). En la mayor parte de los monumentos, las posibles canteras ${ }^{13}$ se localizan en el entorno inmediato de las mámoas. En el sustrato esquistoso predominan las distancias próximas a los $200 \mathrm{~m}$., sin llegar en ningún caso a alcanzar la distancia de $1 \mathrm{~km}$. En la zona migmatítica, la distancia de $1 \mathrm{~km}$. es común casi a las tres cuartas partes de los monumentos. No obstante, las que superan $\operatorname{los} 2 \mathrm{~km}$. representan un grupo relativamente importante (aun-

\footnotetext{
${ }^{12}$ Hecho al que podemos aplicar el mismo comentario que en el caso anterior.

${ }^{13} \mathrm{O}$, cuando menos, los afloramientos rocosos coincidentes con los materiales empleados en la construcción de los ortostatos.
}

"CUADERNOS DE ESTUDIOS GALLEGOS", Tomo XLI, Fascículo 106, Santiago 1993-94. 
que, en todo caso, no llegan a superar el $6 \%$ de los monumentos registrados) por lo que, en ocasiones, otros factores debieron pesar más en la selección del lugar de emplazamiento que el sustrato geológico, ya que en esos casos fué preciso el desplazamiento de las losas graníticas desde distancias superiores a $1 \mathrm{~km}$. y por lugares de orografía dificultosa para el transporte.

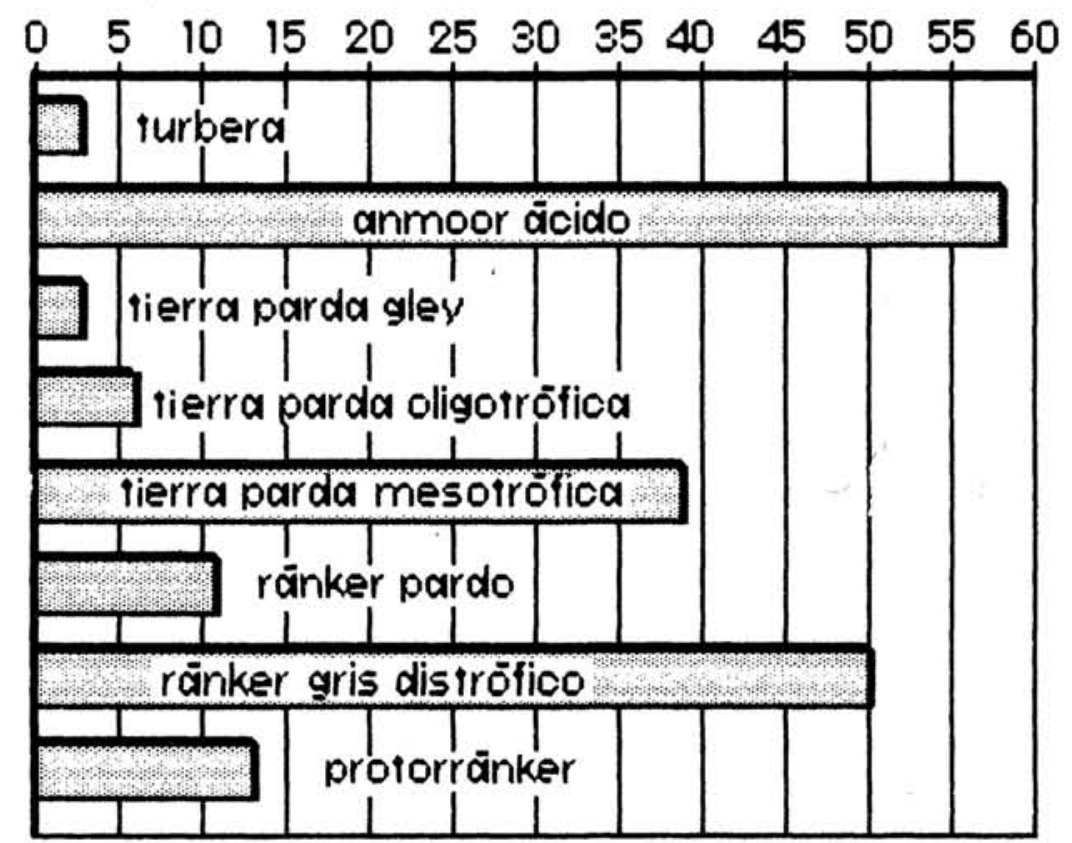

\section{Mämoas y suelos}

Fig. 6.- Cuantificación de las mámoas catalogadas sobre los distintos suelos naturales de A Baixa Limia.

\subsection{Sobre los suelos y su capacidad productiva}

La dispersión o agrupación muy puntual de mámoas, se evidencia sobre suelos ${ }^{14}$ pobres para la actividad económica (rankers), sea ésta agrícola 0 , si bien en menor medida, ganadera (Fig. 6). Así, la edificación de

${ }^{14}$ Empleamos la clasificación de F. Guitián y T. Carballas (1982) por ser la única que, por el momento, ofrece el mapa de suelos de la provincia de Ourense.

"CUADERNOS DE ESTUdioS GALLEGOS", Tomo XLI, Fascículo 106, Santiago 1993-94. 
monumentos en estos lugares, debe estar relacionada con otros condicionantes cuando no se emplazan esas mámoas en las proximidades/ transición hacia tierras más propicias para la explotación agrícola, pecuaria o agropecuaria. Sobre las "áreas de predominio" de los roquedos de silicatos, totalmente estériles, no localizamos ningún monumento. Por otra parte, en la comarca se constata una regularidad y concentración de los emplazamientos sobre dos tipos de suelos con potenciales económicos antagónicos: las tierras pardas, ampliamente cultivadas hoy en día, y los anmoores ácidos, idóneos para la formación de praderas naturales. En los primeros, las tierras pardas, sería factible la compatibilidad de los cultivos con las praderas. Sin embargo, en la zona de anmoores ácidos, los pastizales son genéricamente incompatibles con la actividad agrícola. Ello se debe a varias razones, como la pobreza nutritiva, la escasa potencia de los horizontes, la excesiva humedad del suelo y la altitud, con medias próximas a los $1.200 \mathrm{~m} .{ }^{15}$. Pero sobre los suelos de tierra parda mesotrófica, existen otros condicionantes ajenos a la categoría y distribución del propio suelo, puesto que otras amplias "áreas de predominio" del mismo tipo de tierra parda, se extienden por cotas más bajas que el Valle del Salas ${ }^{16}$, carecen de monumentos tumulares, a no ser microtopónimos como Val de Arca, en Farnadeiros (Muíños).

De esta forma, una de las grandes concentraciones de monumentos tumulares que registramos, la del Valle del Salas Medio, se agrupa en un lugar de cultivo intensivo actual ${ }^{17}$, si bien hoy en día se ve muy limitado por causa de la inundación causada por el embalse de Salas. Otra zona que acoge una elevada densidad de mámoas, la Serra de Leboreiro y sus estribaciones, muestra una ausencia de cultivos practicamente total ${ }^{18}$. Las concentraciones de menor magnitud las registramos en lugares que mantienen distancias desiguales con respecto a los aprovechamientos actuales, si bien es de destacar el predominio de las inferiores a $500 \mathrm{~m}$., y los

\footnotetext{
${ }^{15}$ Las elevadas precipitaciones invernales, alternadas con las heladas, se transforman en nieve durante gran parte del invierno, prolongando desde la primavera el encharcamiento del horizonte $\mathrm{B}$.

${ }^{16}$ Como el entorno del embalse de As Conchas y las vegas de Aceredo-Buscalque y Riocaldo.

17 "Mapa de Cultivos y Aprovechamientos". Ministerio de Agricultura, Pesca y Alimentación, escala 1:50.000.

${ }^{18}$ Con la excepción de una reducida área en torno a la aldea de A Fraga (Lobeira), con una distancia media de $1.000 \mathrm{~m}$. hasta los monumentos más próximos.
}

"CUADERNOS DE ESTUDIOS GALLEGOS", Tomo XLI, Fascículo 106, Santiago 1993-94. 
emplazamientos de los monumentos aislados se caracterizan por las distancias variables, que oscilan entre los $2.500 / 3.000 \mathrm{~m}$. y la inmediatez entre monumentos y explotaciones (Fig. 7).

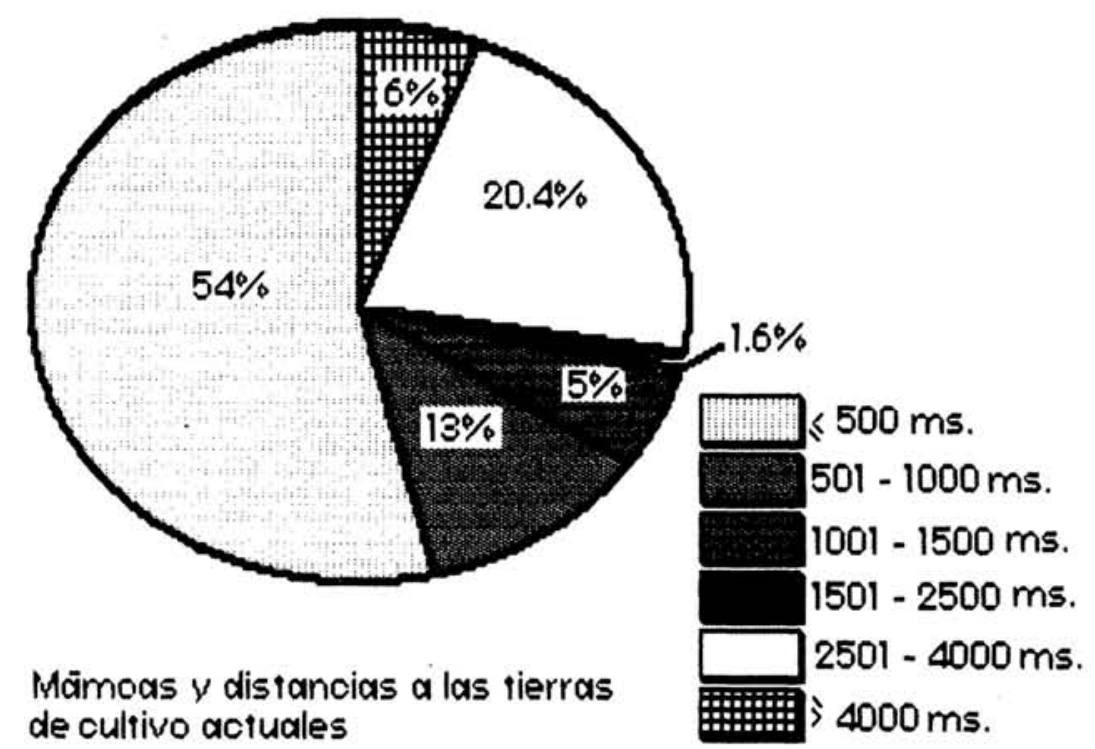

Fig. 7.- Porcentaje de las mámoas catalogadas en función de las distancias hasta los cultivos actuales.

Con respecto a la capacidad productiva de los suelos (Díaz-Fierros Viqueira y Gil Sotres, 1984) ${ }^{19}$, la relación entre las mámoas y aquellos con buena capacidad para las prácticas agrícolas, es mayor que la que inicialmente reflejan los mapas, salvo algunas excepciones. Una de ellas ocurre en la Serra de Leboreiro y sus estribaciones inmediatas, así como en la zona de $\mathrm{O}$ Vieiro, donde las características híbricas ofrecen peores condiciones para los cultivos que las consideradas en el mapa.

Localizamos una de las concentraciones de monumentos en una de las áreas con buenas características para las prácticas agrícolas. De esta for-

\footnotetext{
${ }^{19}$ No queremos dejar de reseñar que aún no fueron editados por el Ministerio de Agricultura las hojas de los mapas de "Clases Agrobiológicas (Plan de Evaluación de Recursos Agrarios)" correspondientes con la comarca de A Baixa Limia.
}

"CUADERNOS DE ESTUdIOS GALLEGOS", Tomo XLI, Fascículo 106, Santiago 1993-94. 
ma, las tierras de Clases A, B e C del Valle del Salas Medio y Alto, así como del nacimiento del río de Ponte Maior, acogen una de las grandes concentraciones tumulares de la comarca. Otra de las grandes concentraciones de monumentos tiene lugar sobre las tierras de Clases F y G (Serra de Leboreiro y estribaciones) con malas cualidades para las actividades agrícolas, si bien idóneas como praderas naturales.

Sin embargo, la distribución de mámoas es desigual y más irregular en las zonas con capacidades productivas intermedias. Los peores sectores productivos de A Baixa Limia, donde se combina el predominio de las Clases $\mathrm{F}$ y $\mathrm{G}$ con altos porcentajes de afloramientos rocosos o con pendientes de valores también altos, muestran una total ausencia de emplazamientos tumulares.

Podemos observar como existe una muy alta correlación entre los mapas de capacidad productiva de los suelos, el de cultivos y aprovechamientos actuales y el edafológico en relación con los porcentajes de monumentos tumulares repartidos sobre las distintas categorías y zonas productivas.

\section{BIBLIOGRAFÍA CITADA}

BARROS SIVELO, R., 1875: Antigüedades de Galicia. A Coruña.

COUCEIRO FREIJOMIL, A., 1937: "Monumentos de la provincia de Orense. Descripción histórica y bibliográfica". Boletín de la Comisión de Monumentos de Orense, XI, Ourense, pp. 217-241-265-289-337387-435.

DÍAZ-FIERROS VIQUEIRA, F. y GIL SOTRES, F., 1984: Capacidad productiva de los suelos de Galicia. Mapa 1:200.000. Universidad de Santiago de Compostela, Santiago.

DÍAZ SANJURJO, M., 1904: "Los caminos antiguos y el itinerario número 18 de Antonino en la provincia de Orense". Boletín de la Comisión de Monumentos de Orense, II, Ourense, pp. 229-340.

EGUILETA FRANCO, J.M., 1986: Aportación ao estudo dos contextos megalíticos galaicos; os axuares megalíticos da Galicia Sur: o Miño Medio e a Limia. Tesis de Licenciatura inédita, Universidad de Santia- 
go, Facultad de Geografía e Historia, Santiago de Compostela. 1987. "Catálogo dos materiais ergolóxicos depositados no Museo de Ourense procedentes de túmulos prehistóricos". Boletín Auriense, XVII, Ourense, pp. 9-98.

1991. "Arqueoloxía na Baixa Limia: o concello de Muíños e o seu marco arqueo-xeográfico (Campaña 1990)". Arqueoloxía/Informes 4 (en prensa).

1994. Megalitismo e Calcolítico na Baixa Limia galega. Tesis Doctoral microfilmada. Universidad de Santiago de Compostela.

EGUILETA FRANCO, J.M., RODRÍGUEZ CAO, C. y XUSTO RODRÍGUEZ, M.: 1991. "Arqueoloxía na Baixa Limia: o encoro de Lindoso e o seu medio histórico (Lobios, Ourense)". Arqueoloxía/Informes 2. Campaña 1988, Santiago de Compostela, pp. 139-156.

FABREGAS VALCARCE, R.: 1992. Megalitismo del noroeste de la Península Ibérica. Tipología y secuencia de los materiales líticos. "Aula Abierta", 58, Universidad Nacional de Educación a Distancia, Madrid.

FABREGAS VALCARCE, R. y FUENTE ANDRÉS, F. de la: 1988. Aproximaciones a la cultura material del megalitismo gallego: la industria lítica pulimentada y el material cerámico. "Arqueohistoria", 2. Santiago de Compostela.

FERNÁNDEZ ALONSO, B.: 1879. El río Limia. Propaganda Gallega. Ourense.

1906. "Galicia Prehistórica". Boletín de la Comisión de Monumentos de Orense, III, 65, Ourense, pp. 327-332.

FERRO COUSELO, X.: 1972. "Reseña del Museo". Boletín Auriense, II, Ourense, pp. 319-345.

FORTES, J.: 1901. "A necrópole dolménica de Salles (Terras de Barroso)". Portugalia, I, Porto, pp. 665-686.

GARCÍA, H.: 1922. "Monte de Santa Eufemia, ¿Antigua Obóbriga?". Boletín de la Comisión de Monumentos de Orense, VI, Ourense, pp. 499-502.

"CUADERNOS DE ESTUdios GALLEGOS", Tomo XLI, Fascículo 106, Santiago 1993-94. 
GUITIÁN OJEA, F. y CARBALLAS, T.: 1982. Suelos naturales de la provincia de Orense. Consejo Superior de Investigaciones Científicas, Instituto de Investigaciones Agrobiológicas de Galicia, Santiago de Compostela.

LEISNER, G.: 1938. Verbreitung und Typologie der GalizishNordportugiesischen Megalithgräber. Marburg.

LEISNER, G. y LEISNER, V.: 1959. Die Megalithgräber der Iberischen Halbinsel. Der Westen. Berlin.

LÓPEZ CUEVILLAS, F.: 1923. "Os dólmenes do Monte das Motas en Lobeira". Nós, 18, Santiago de Compostela, pp. 18-21.

1925a. "As mámoas do concello de Lobeira". Boletín de la Real Academia Gallega, XV, A Coruña, pp. 25-39.

1925b. "As mámoas do concello de Rairiz". Boletín de la Real Academia Gallega, XV, A Coruña, pp. 235-245.

1927a. "Dúas estacións dolménicas. As mamoiñas da Serra da Moa e as antas de Maus de Salas". Nós, Fasc. 44, Santiago de Compostela, pp. $5-10$.

1927 b. "Papeletas para un inventario das mámoas galegas". Nós, Fasc. 39 y 40, Santiago de Compostela, pp. 8-13 y 9-13.

1930. "Novas cerámicas das antas galegas". Sociedade Portuguesa de Antropología e Etnología, IV, Porto, pp. 263-282.

1948. "La Cultura Megalítica del NO peninsular". Boletín del Museo Arqueológico Provincial de Orense, IV, Ourense, pp. 3-19.

1955. "Caracteres de la cultura megalítica del Noroeste". Actas del III Congreso Nacional de Arqueología, (Santiago 1953), Zaragoza, pp. 262-272.

1959. "La época megalítica en el Noroeste de la Península". Caesaraugusta, 12/14, Zaragoza, pp. 21-77.

1973. "A Edade Megalítica". En OTERO PEDRAYO, R. (Dir.), Historia de Galicia, III, (Prehistoria), (original redactado en 1952), Buenos Aires.

LÓPEZ CUEVILLAS, F. y BOUZA BREY, F.: 1931. "La Civilización Neo-eneolítica gallega". Archivo Español de Arte y Arqueología, VII, Madrid, pp. 41-61. 
LÓPEZ CUEVILLAS, F. y LORENZO FERNÁNDEZ, X.: 1930. Vila de Calvos. Notas etnográficas e folklóricas. "Publicaciones do Seminario de Estudos Galegos", V, Santiago de Compostela.

1958. "Dos necrópolis doménicas". Cuadernos de Estudios Gallegos, 41, Santiago de Compostela, pp. 285-291.

LORENZO, X. y FARIÑA, L.: 1933. "Tres estacións de Arte Rupestre na Serra de Leboreiro". Nós, V, Santiago de Compostela, pp. 124-132.

OBERMAIER, H.: 1923. "Impresiones de un viaje prehistórico por Galicia". Boletín de la Comisión de Monumentos de Ourense, VII, Ourense, pp. 1-25.

OLIVEIRA JORGE, V.: 1982. Megalitismo do norte de Portugal. Os monumentos e a súa problemática no contexto europeu. Dissertaçao de Doutoramento policopiada, Facultade de Letras do Porto, Porto.

RODRÍGUEZ CAO, C.: 1991. "Prospección arqueolóxica da comarca de Bande (Ourense)". Arqueoloxía Informes 2. Campaña 1988, Xunta de Galicia, Santiago de Compostela, pp. 237-240.

1993. "Nuevas aportaciones al arte megalítico. La Mota Grande (Verea)". Boletin Auriense, XXIII, Ourense, pp. 9-19.

RODRÍGUEZ COMENERO, A.: 1971. "La cultura megalítica en el Alto Bubal". Boletín Auriense, I, Ourense, pp. 31-60.

TABOADA CHIVITE, X.: 1973. "Addenta et corrigenda. A investigación arqueolóxica desde 1952". En OTERO PEDRAYO, R. (Dir.), Historia de Galicia, III, (Prehistoria), (original redactado en 1952), Buenos Aires.

VÁZQUEZ NÚÑEZ, A.: 1901. "Estudios Protohistóricos, Las mámoas". Boletín de la Comisión de Monumentos de Orense, III, Ourense, pp. 345-353.

VV.AA.: 1980. "Ocupación e poboamento na comarca de Bande: o Val do Salas". Comunicación presentada ó II Seminario de Arqueoloxía do NO Peninsular, Santiago de Compostela.

"CUADERNOS DE ESTUDIOS GALLEGOS", Tomo XLI, Fascículo 106, Santiago 1993-94. 